\title{
An overlapping case of in situ mantle cell neoplasia and leukemic non-nodal mantle cell lymphoma
}

\author{
Ryota Matsuoka, ${ }^{1)}$ Noriaki Sakamoto, ${ }^{1)}$ Mamiko Sakata-Yanagimoto, ${ }^{2)}$ Shigeru Chiba, ${ }^{2)}$ \\ Masayuki Noguchi, ${ }^{1)}$ Naoya Nakamura ${ }^{3)}$
}

In situ mantle cell neoplasia (isMCN) and leukemic non-nodal mantle cell lymphoma (nnMCL) are classified as an indolent subtype of mantle cell lymphoma (MCL). The tumor cells of isMCN are restricted to the inner layer of the lymphoid tissue mantle zone, exhibiting an in situ pattern histologically. On the other hand, nnMCL is distributed in the peripheral blood, bone marrow and sometimes the spleen, but lymphadenopathy or systemic organ involvement is rare. We report a case of isMCN in a submandibular lymph node resected from a 65-year-old Japanese male. The tumor cells were positive for cyclin D1 (CCND1) and SOX11 expression, and were restricted to the mantle zone area of the lymph node. However, tumor cells were also detected in the stomach mucosa, bone marrow tissue and peripheral blood, suggesting nnMCL. isMCN and nnMCL may have a partly overlapping disease spectrum, although the correlation between these two subtypes has not been well described. This present case demonstrated characteristics overlapping between isMCN and nnMCL.

Keywords: in situ mantle cell neoplasia, leukemic non-nodal mantle cell lymphoma, cyclin D1, SOX11

\section{INTRODUCTION}

Mantle cell lymphoma (MCL) is an aggressive B-cell neoplasm characterized by a translocation involving chromosomes 11 and 14, t(11;14)(q13;q32), which juxtaposes the cyclin D1 (CCND1) oncogene to the immunoglobulin heavy chain (IGH) locus. As a result, the tumor cells overexpress CCND1 protein, which leads to tumor proliferation. ${ }^{1,2}$ The tumor cells are usually positive for SOX11 expression. Most cases are diagnosed at an advanced stage and the overall survival rate is 3-5 years. ${ }^{3,4}$

MCL exhibits varying histological patterns such as the nodular, diffuse and mantle zone patterns. In rare cases, CCND1-positive tumor cells remain restricted to the inner layer of the mantle zone and exhibit indolent behavior with long-term survival even without treatment. ${ }^{5-8}$ This type of MCL was newly defined as in situ mantle cell neoplasia (isMCN) in the 2017 WHO classification. ${ }^{9}$ Although an in situ distribution of tumor cells may be found in conventional MCL (cMCL) as part of the histological pattern, isMCN demonstrating a solely in situ pattern is rare and most cases are incidentally found. ${ }^{8}$ Of note, this subtype is defined only by the histological location of the tumor cells in lymphoid tissue and systemic distribution of the tumor cells will not exclude the diagnosis. ${ }^{9}$

On the other hand, there are many studies in the last decade reporting cases of MCL with leukemic forms having an indolent clinical course, and the affected patients survived without treatment. ${ }^{10-14}$ This type of MCL was newly defined as leukemic non-nodal-type MCL (nnMCL) in the 2017 WHO classification. ${ }^{9}$ Patients with nnMCL present with peripheral blood, bone marrow and splenic involvement of the tumor cells, without significant lymphadenopathy or systemic organ involvement. The tumor cells are positive for CCND1 and negative for SOX11.7,12,14 isMCN and nnMCL may have a partly overlapping disease spectrum, although the correlation between these two subtypes has not been well described.

We report a case of isMCN in which the distribution of the tumor cells was restricted to the inner layer of the mantle zone, but some cells were also distributed systemically. The tumor cells were positive for both CCND1 and SOX11 expression. The relationship between isMCN and nnMCL is discussed.

\footnotetext{
Received: May 26, 2020. Revised: July 24, 2020. Accepted: August 2, 2020. J-STAGE Advance Published: October 8, 2020

DOI:10.3960/jslrt.20022

${ }^{1}$ Department of Diagnostic Pathology, Faculty of Medicine, University of Tsukuba, Ibaraki, Japan, ${ }^{2}$ Department of Hematology, Faculty of Medicine, University of Tsukuba, Ibaraki, Japan, ${ }^{3}$ Department of Pathology, Tokai University School of Medicine, Kanagawa, Japan

Corresponding author: Noriaki Sakamoto, MD, Department of Diagnostic Pathology, Faculty of Medicine, University of Tsukuba, 2-1-1 Amakubo, Tsukuba-shi, Ibaraki 305-8576,

Japan. E-mail: n.sakamoto@md.tsukuba.ac.jp

Copyright (C) 2020 The Japanese Society for Lymphoreticular Tissue Research

(cc) BY-NC-SA This work is licensed under a Creative Commons Attribution-NonCommercial-ShareAlike 4.0 International License.
} 


\section{CASE PRESENTATION}

The patient was a 65 -year-old Japanese male who presented to a local hospital with a 5-year history of slowly increasing submandibular lymph node swelling. He had had no B-symptoms (fever, night sweats and weight loss). A complete peripheral blood count revealed a white blood cell count of $4,900 / \mu \mathrm{L}$ (neutrophils: $58.0 \%$, lymphocytes: $32.0 \%$, monocytes: $5.0 \%$, eosinophils: $4.5 \%$, basophils: $0.5 \%$ ), hemoglobin of $15.3 \mathrm{~g} / \mathrm{dL}$, platelet count of $29.7 \times 10^{4} / \mu \mathrm{L}$, lactate dehydrogenase level of $164 \mathrm{U} / \mathrm{L}$ and soluble interleukin-2 receptor level of $232 \mathrm{U} / \mathrm{L}$. Positron emission tomography-CT demonstrated increased glucose metabolism and standardized uptake values only in the submandibular lymph node. The size of the lymph node was approximately $1.8 \mathrm{~cm}$ and excisional lymph node biopsy was performed for pathological diagnosis, but neither cytogenetic nor flow cytometry analysis was performed. Histologically, the excised lymph node demonstrated multiple hyperplastic lymph follicles with reactive germinal centers (Figure 1a). The mantle zone was narrow. Small to mid-sized lymphocytes were present in the mantle zone. Immunohistochemistry demonstrated CCND1 positivity in lymphocytes restricted to the mantle zone area. These lymphocytes were also positive for CD20, bcl-2 and SOX11, and negative for CD3, CD5, CD10, bcl-6 and pSTAT3, and exhibited a low Ki-67 labeling index (Figure 1b-f). Fluorescence in situ hybridization (FISH) analysis demonstrated rearrangement of $I G H / C C N D 1$ (Figure 1g), yielding a diagnosis of isMCN.

The patient was referred to University of Tsukuba Hospital for further studies. A systemic search using gastroendoscopy revealed a small erosion in the stomach, which was biopsied. Histological examination of the biopsy sample demonstrated small lymphocytes aggregated in the gastric mucosa. These were positive for CD20, CCND1 and SOX11, negative for $\mathrm{CD} 5$ and positive for rearrangement of $I G H / C C N D 1$ (Figure 2). Bone marrow biopsy also demonstrated slight infiltration of B-cells that were positive for CCND1 and SOX11, negative for CD5 and positive for rearrangement of $I G H / C C N D 1$ (Figure 3). Flow cytometry analysis of the peripheral blood revealed a population of B-cells ( $40 \%$ of leucocytes) within the lymphocyte gate (CD45/side scatter). Although CD5 immunohistochemistry was negative for tumor cells in the lymph node, stomach and bone marrow, the B-cells in the peripheral blood expressed CD5 and kappa light chain according to flow cytometry (Figure 4a). FISH analysis of peripheral blood also demonstrated rearrangement of $I G H / C C N D 1$ (Figure 4b). These findings from the stomach, bone marrow and peripheral blood suggested that the isMCN tumor cells were distributed systemically. The immunoglobulin heavy chain variable region $(I G H V)$ gene was analyzed by PCR amplification and Sanger sequencing using DNA from the bone marrow sample, and subjected to an IGBlast search (https://www.ncbi. nlm.nih.gov/igblast/). The mutation rate was $5.6 \% \mathrm{com}$ pared with the germline $I G H V$ gene; therefore, based on previous reports, we concluded that these tumor cells were hypermutated. ${ }^{15,16}$

The patient was followed up closely without treatment and there has been no evidence of recurrence (follow-up time: 40 months).

\section{DISCUSSION}

The present case meets the criteria for lymph node isMCN. We also found tumor cells that demonstrated
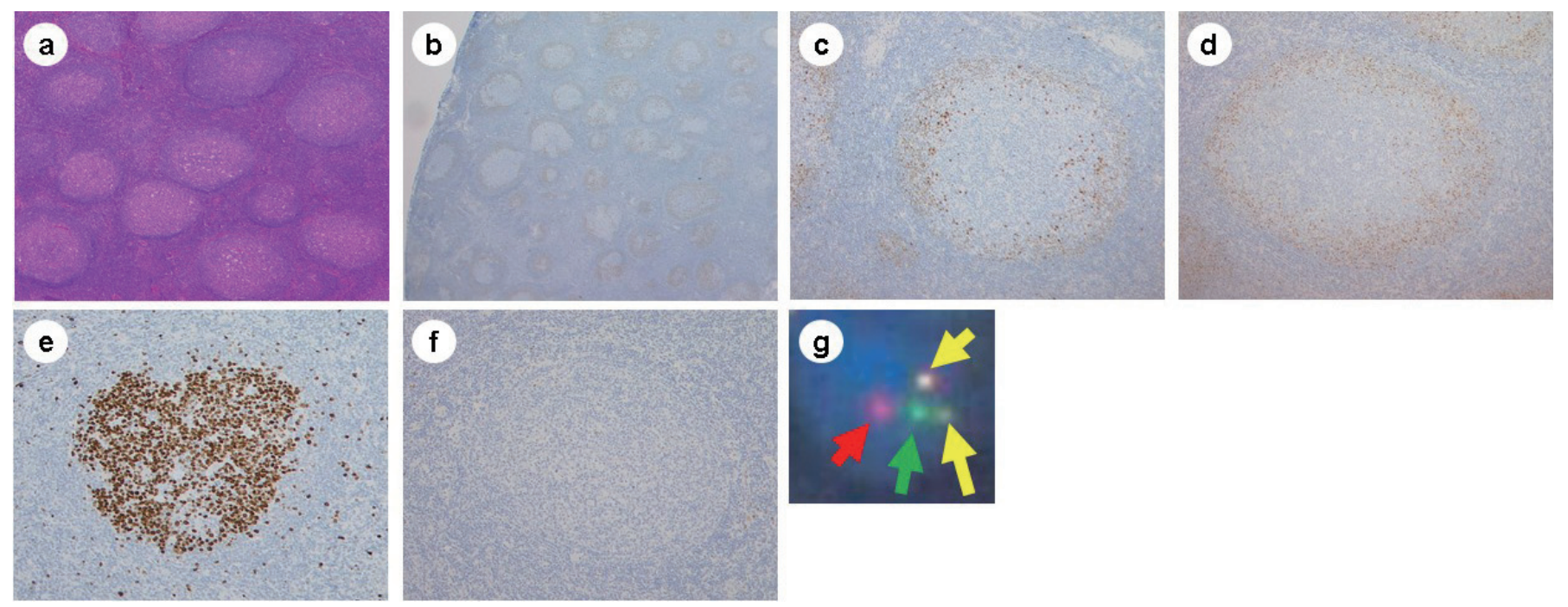

Fig. 1. Lymph node. (a) H\&E staining (x40) shows many lymph follicles with expanded germinal centers and narrow mantle zones. (b, $\boldsymbol{c}$ ) Cyclin D1 (CCND1) staining (b: x12.5, c: x100) shows that dense CCND1- positive cells are restricted to the inner layer of the mantle zone. The mantle zones are independent of each other and there is no evidence of mantle cell lymphoma with a mantle zone growth pattern. $(\boldsymbol{d})$ SOX11 staining (x100) exhibits a pattern similar to that of CCND1 staining. (e) Ki-67 staining (x100) shows that the inner layer of the mantle zone has a low proliferation rate. $(f)$ pSTAT3 staining (x100) is negative in the inner layer of the mantle zone. ( $g$ ) Fluorescence in situ hybridization (FISH) analysis using a dual- fusion probe for $C C N D 1$ and the $I G H$ locus shows two yellow signals, one red signal and one green signal, indicative of $\mathrm{t}(11 ; 14)$ [red: CCND1 (11q13), green: IGH (14q32), yellow: fusion signal]. 

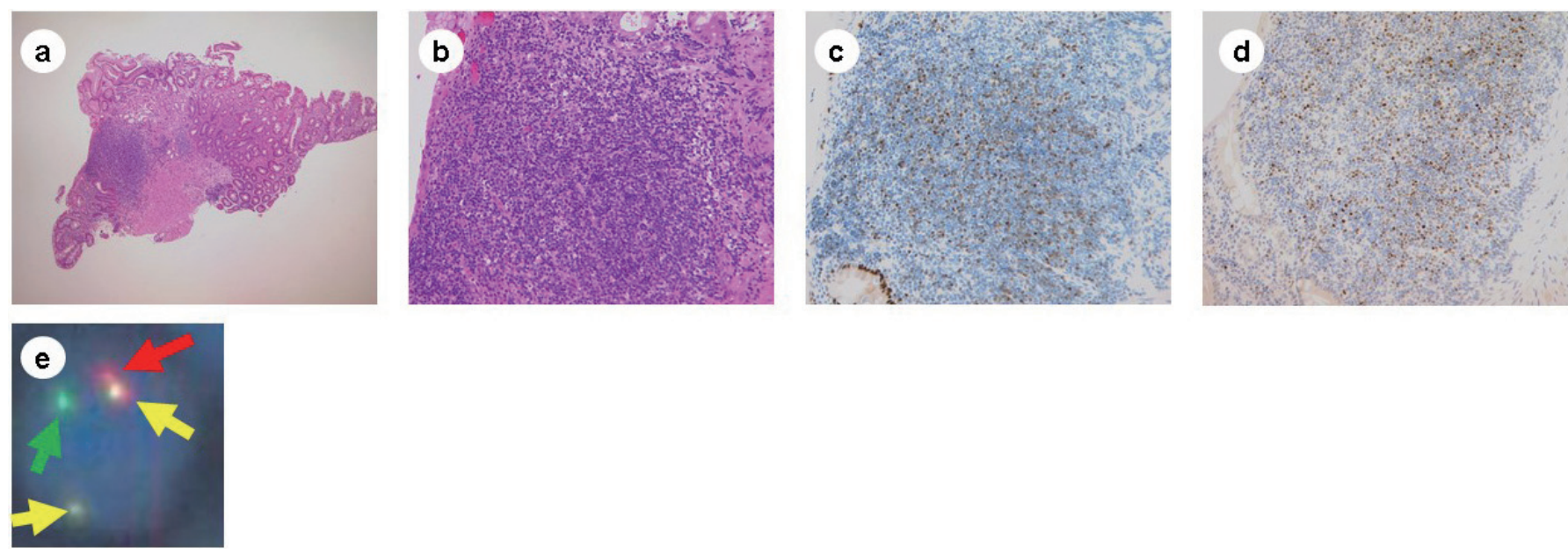

Fig. 2. Stomach. $(\boldsymbol{a}, \boldsymbol{b}) \mathrm{H} \& \mathrm{E}$ staining (a: x12.5, b: x100) shows small lymphocyte aggregates in the mucosa. (c) Cyclin D1 (CCND1) staining (x400) reveals numerous CCND1-positive lymphocytes in the mucosa. (d) SOX11 staining (x100) exhibits a pattern similar to that of CCND1 staining. (e) FISH analysis using a dual-fusion probe for $C C N D I$ and the $I G H$ locus shows two yellow signals, one red signal and one green signal, indicative of $\mathrm{t}(11 ; 14)$ [red: $C C N D 1(11 \mathrm{q} 13)$, green: $I G H(14 \mathrm{q} 32)$, yellow: fusion signal].
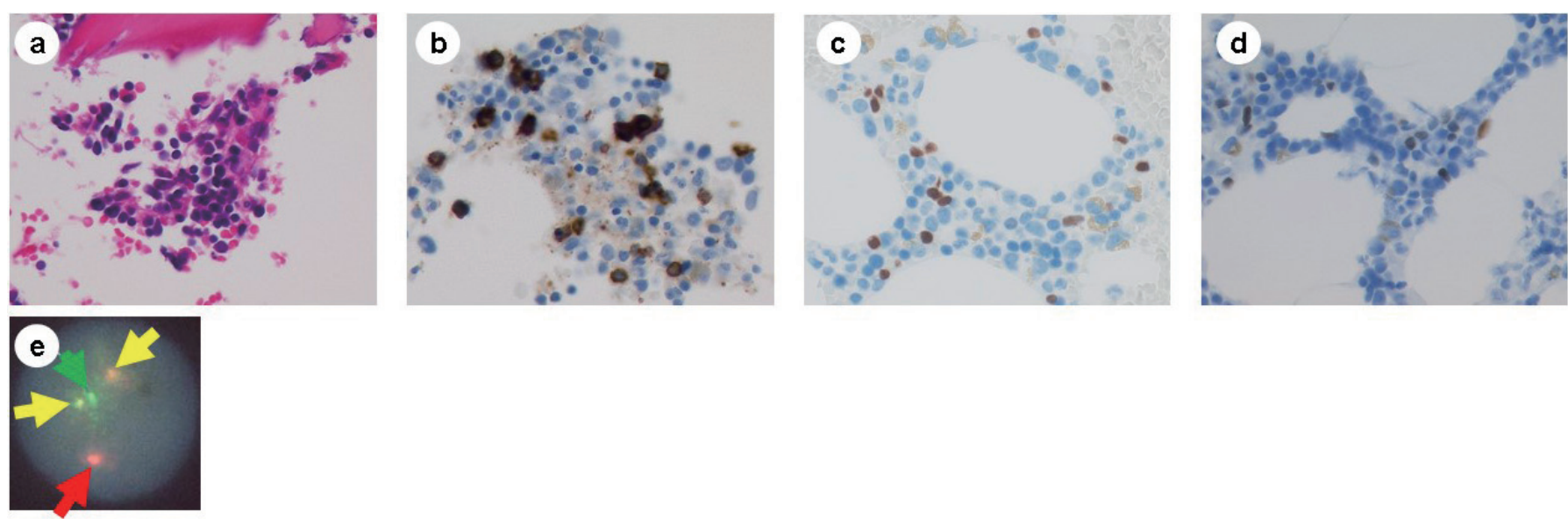

Fig. 3. Bone marrow. (a) H\&E staining (x400) and (b) CD20 staining (x400) show scattered small cells. (c) Cyclin D1 (CCND1) staining (x400) reveals scattered positivity in the bone marrow. (d) SOX11 staining (x100) exhibits a pattern similar to that of CCND1 staining. (e) FISH analysis using a dual-fusion probe for $C C N D I$ and the $I G H$ locus shows two yellow signals, one red signal and one green signal, indicative of $\mathrm{t}(11 ; 14)$ [red: $C C N D 1$ (11q13), green: $I G H(14 \mathrm{q} 32)$, yellow: fusion signal].

a
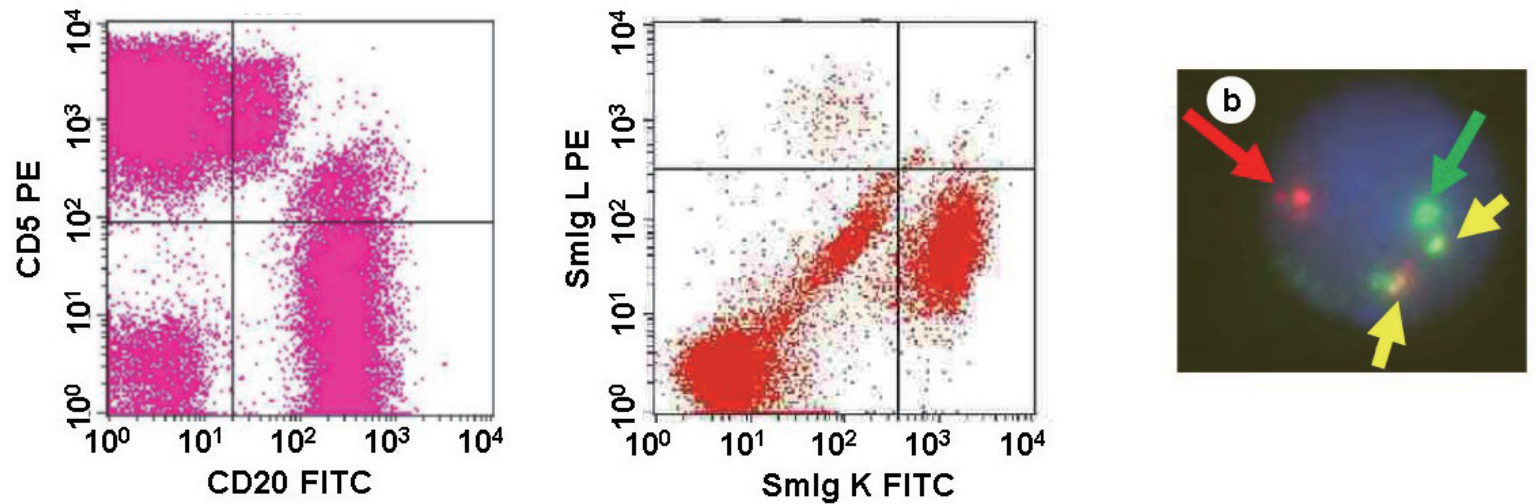

Fig. 4. Peripheral blood. (a) Flow cytometry revealed that CD20- and CD5-positive B-cells displayed surface immunoglobulin kappa light chain restriction. (b) FISH analysis using a dual-fusion probe for cyclinD1 (CCNDI) and the $I G H$ locus shows two yellow signals, one red signal and one green signal, indicative of $\mathrm{t}(11 ; 14)$ [red: CCND1 (11q13), green: $I G H$ (14q32), yellow: fusion signal]. 
similar immunohistochemistry and FISH patterns with the is $\mathrm{MCN}$ in the stomach, bone marrow tissue and peripheral blood, resembling lymphoma with leukemic involvement. As patients with conventional MCL (cMCL) often exhibit leukemic involvement and have a poor prognosis, ${ }^{17,18}$ detection of cMCL tumor cells in the bone marrow or peripheral blood is important. nnMCL, on the other hand, follows an indolent clinical course despite its leukemic form. Although each concept of isMCL and nnMCL has been independently constructed, a recent case series suggested that isMCN overlaps with nnMCL. Carvajal-Cuenca et al. reported that in 5 of 23 cases $(21.7 \%)$ of isMCN, the tumor cells involved the bone marrow and/or peripheral blood. Although nnMCL is usually negative for SOX11, one case in the latter series was positive. ${ }^{5}$ In addition, Espinet et al. reported a case series of nnMCL in which 5 of 13 cases (38.5\%) had CCND1-positive tumor cells in secondary lymphoid tissue, being located mainly in the mantle zone and displaying an in situ pattern. ${ }^{14}$ All of these 5 cases were SOX11-negative. However, neither of the latter two case series included an analysis of somatic hypermutation.

Thus, unlike epithelial in situ carcinoma, the tumor cells of isMCN may spread systemically despite having an "in situ" status, as observed in our case. This suggests that, based on the WHO classification definition of isMCN and nnMCL, these two tumor subtypes overlap in some cases. In contrast, 1 out of 12 cases $(8.3 \%)$ in the former case series of isMCN progressed to $\mathrm{CMCL}$, suggesting that the former is a precursor lesion of the latter. ${ }^{5}$ These findings are informative regarding the relationships among $\mathrm{CMCL}, \mathrm{nnMCL}$ and isMCN, and the differences in their biological characteristics.

A recently proposed model of MCL tumorigenesis may help clarify this issue (Figure 5). ${ }^{1}$ In this model, a naïve B-cell harboring the $I G H / C C N D I$ translocation originating from bone marrow reaches the lymph node mantle zone and forms an in situ lesion. Most of the subsequently developing tumor cells may then progress to cMCL with no $I G H V$ somatic hypermutations, suggesting that they do not transit

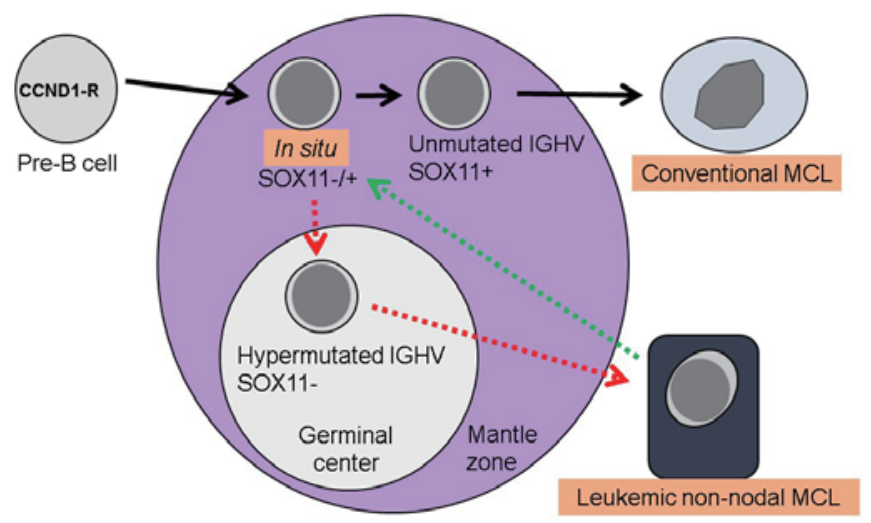

Fig. 5. Hypothetical model of two different molecular subtypes of MCL. isMCN may be the precursor lesion of nnMCL (red dotted arrows), or nnMCL tumor cells will home in on the mantle zone area (green dotted arrow).

CCND1-R: cyclin D1-rearrangement, IGHV: immunoglobulin heavy chain variable region gene, MCL: mantle cell lymphoma through the germinal center. These tumor cells have other features such as SOX11 expression, genetic instability and a high number of genomic aberrations. On the other hand, some tumor cells may pass through the germinal center, spread through the peripheral blood and become nnMCL, which often has $I G H V$ somatic hypermutations and does not express SOX11.

In this model of MCL tumorigenesis, although isMCN is thought to be a precursor lesion of cMCL, it can also be a precursor lesion of nnMCL (Figure 5, red dotted arrows), or nnMCL tumor cells will home in on the mantle zone area of lymphoid tissue and exhibit an in situ pattern (Figure 5, green dotted arrow), as demonstrated in the case series described above. cMCL often exhibits SOX11+/IGHV non-mutation, whereas nnMCL exhibits SOX11-/IGHV hypermutation, although expression of SOX11 and somatic hypermutation status in isMCN is not well known. In terms of the histology, progression pattern and somatic hypermutation status, the present case met the criteria for the indolent type of MCL, although the tumor cells expressed SOX11. In a recent case series of MCL, 5 out of 26 cases (19\%) demonstrated $I G H V$ hypermutation, and $I G H V$ was significantly mutated in 11 out of 26 cases (42\%), which also expressed SOX11. ${ }^{19}$ Although this series lacked histological descriptions and the histology of SOX11+ with IGHV hypermutated/ significantly mutated MCL was unclear, some cases of MCL demonstrated discrepancy between somatic hypermutation and SOX11 expression, as observed in our present case.

SOX11 is a neuronal transcription factor thought to be important for the progression of MCL and is highly expressed in MCL (80-90\% of cases). ${ }^{20}$ Recent reports stated that the expression of SOX11 in MCL is an independent prognostic factor correlated with a lack of somatic hypermutation or a low mutation status. ${ }^{19,21}$ However, the function of SOX11 in MCL is unclear. Through an examination of MCL cell lines and patient-derived xenografts, Mohanty et al. revealed that the SOX11-negative indolent subtype of MCL is related to upregulation of STAT3..$^{22}$ In addition, a recent report found that STAT3 was activated in the majority $(70 \%)$ of indolent leukemic MCL. ${ }^{23}$ These reports suggest that STAT3 plays a key role in repressing SOX11 expression and thus accounts for the indolent course of SOX11-negative MCL. Consistent with the hypothesis that activated STAT3 represses SOX11, our present case, which was SOX11-positive, was negative for pSTAT3 immunohistochemistry (Figure 1f).

We reported a case of isMCN with systemic involvement that overlapped with nnMCL. Although isMCN has a favorable prognosis and progression to conventional MCL is rare, the patient in the present case will require careful observation due to SOX11 expression. As isMCN is a rare variant with few examples of leukemic change, its biological features and prognosis will require further study.

\section{CONFLICT OF INTEREST}

The authors have no conflicts to disclose. 


\section{REFERENCES}

1 Jares P, Colomer D, Campo E. Molecular pathogenesis of mantle cell lymphoma. J Clin Invest. 2012; 122 : 3416-3423.

2 Beà S. Cyclin D1 transcriptional activation in MCL. Blood. 2014; $123: 1979-1980$.

3 Martin P, Chadburn A, Christos P, et al. Outcome of deferred initial therapy in mantle-cell lymphoma. J Clin Oncol. 2009; 27 : 1209-1213.

4 Herrmann A, Hoster E, Zwingers T, et al. Improvement of overall survival in advanced stage mantle cell lymphoma. J Clin Oncol. 2009; 27 : 511-518.

5 Carvajal-Cuenca A, Sua LF, Silva NM, et al. In situ mantle cell lymphoma: clinical implications of an incidental finding with indolent clinical behavior. Haematologica. 2012; 97 : 270-278.

6 Hsu P, Yang T, Sheikh-Fayyaz S, et al. Mantle cell lymphoma with in situ or mantle zone growth pattern: a study of five cases and review of literature. Int J Clin Exp Pathol. 2014; 7 : 1042-1050.

7 Ye H, Desai A, Zeng D, et al. Smoldering mantle cell lymphoma. J Exp Clin Cancer Res. 2017; $36: 185$.

8 Adam P, Schiefer AI, Prill S, et al. Incidence of preclinical manifestations of mantle cell lymphoma and mantle cell lymphoma in situ in reactive lymphoid tissues. Mod Pathol. 2012; 25 : 1629-1636.

9 Swerdlow S, Campo E, Harris N, et al. WHO Classification of Tumours of Haematopoietic and Lymphoid Tissues. Revised 4th ed, Lyon, IARC Press. 2017; pp.285-290.

10 Nodit L, Bahler DW, Jacobs SA, Locker J, Swerdlow SH. Indolent mantle cell lymphoma with nodal involvement and mutated immunoglobulin heavy chain genes. Hum Pathol. 2003; $34: 1030-1034$.

11 Orchard J, Garand R, Davis Z, et al. A subset of $\mathrm{t}(11 ; 14)$ lymphoma with mantle cell features displays mutated $\operatorname{IgVH}$ genes and includes patients with good prognosis, nonnodal disease. Blood. 2003; 101 : 4975-4981.

12 Fernàndez V, Salamero $\mathrm{O}$, Espinet $\mathrm{B}$, et al. Genomic and gene expression profiling defines indolent forms of mantle cell lymphoma. Cancer Res. 2010; 70 : 1408-1418.
13 Royo C, Navarro A, Clot G, et al. Non-nodal type of mantle cell lymphoma is a specific biological and clinical subgroup of the disease. Leukemia. 2012; $26: 1895-1898$

14 Espinet B, Ferrer A, Bellosillo B, et al. Distinction between asymptomatic monoclonal B-cell lymphocytosis with cyclin D1 overexpression and mantle cell lymphoma: from molecular profiling to flow cytometry. Clin Cancer Res. 2014; 20: 1007-1019.

15 Hamblin TJ, Davis Z, Gardiner A, Oscier DG, Stevenson FK. Unmutated $\operatorname{Ig~} \mathrm{V}(\mathrm{H})$ genes are associated with a more aggressive form of chronic lymphocytic leukemia. Blood. 1999; 94 : 1848-1854

16 Hadzidimitriou A, Agathangelidis A, Darzentas N, et al. Is there a role for antigen selection in mantle cell lymphoma? Immunogenetic support from a series of 807 cases. Blood. 2011; $118: 3088-3095$.

17 Hoster E, Dreyling M, Klapper W, et al. A new prognostic index (MIPI) for patients with advanced-stage mantle cell lymphoma. Blood. 2008; 111 : 558-565.

18 Ferrer A, Salaverria I, Bosch F, et al. Leukemic involvement is a common feature in mantle cell lymphoma. Cancer. 2007; 109 : 2473-2480.

19 Navarro A, Clot G, Royo C, et al. Molecular subsets of mantle cell lymphoma defined by the IGHV mutational status and SOX11 expression have distinct biologic and clinical features. Cancer Res. 2012; 72 : 5307-5316.

20 Ferrando AA. SOX11 is a mantle cell lymphoma oncogene. Blood. 2013; $121: 2169-2170$.

21 Nygren L, Baumgartner-Wennerholm S, Klimkowska M, et al. Prognostic role of SOX11 in a population-based cohort of mantle cell lymphoma. Blood. 2012; 119 : 4215-4223.

22 Mohanty A, Sandoval N, Phan A, et al. Regulation of SOX11 expression through CCND1 and STAT3 in mantle cell lymphoma. Blood. 2019; $133:$ 306-318.

23 Baran-Marszak F, Boukhiar M, Harel S, et al. Constitutive and B-cell receptor-induced activation of STAT3 are important signaling pathways targeted by bortezomib in leukemic mantle cell lymphoma. Haematologica. 2010; 95 : 1865-1872. 